\title{
Defective food concepts
}

\section{Andrea Borghini $^{1}$ (D) Nicola Piras ${ }^{1} \cdot$ Beatrice Serini $^{1}$}

Received: 15 October 2020 / Accepted: 20 July 2021 / Published online: 2 August 2021

(c) The Author(s) 2021

\begin{abstract}
Our aim in this paper is to employ conceptual negotiation to inform a method of rethinking defective food concepts, that is concepts that fail to suitably represent a certain food-related domain or that offer representations that run counter to the interests of their users. We begin by sorting out four dimensions of a food concept: (i) the data upon which it rests and the methodology by which those data are gathered; (ii) the ontology that sustains it; (iii) the social acts that serve to negotiate and establish the concept; (iv) and the aims and values that it fosters. We then discuss the conditions that make a food concept defective, pointing out four types of defects-fragility, polarization, incoherence, and schizophrenia - which we illustrate by means of two specific examples: local food and healthy food.
\end{abstract}

Keywords Food concepts · Food ontology · Conceptual negotiation · Healthy food · Local food

This article belongs to the topical collection "Concept Formation in the Natural and Social Sciences: Empirical and Normative Aspects", edited by Georg Brun, Catherine Herfeld, and Kevin Reuter.

Andrea Borghini

andrea.borghini@unimi.it

Nicola Piras

nicola.piras@unimi.it

Beatrice Serini

beatrice.serini@unimi.it

1 Department of Philosophy "Piero Martinetti”, University of Milan, via Festa del Perdono 7, 20122 Milano, Italy 


\section{Introduction}

Boxes of cornflakes typically sold in supermarkets advertise their content through a repertoire of concepts expressed by recognizable tags_like "zero-fat," "crispy and golden," "the original breakfast," "high in iron," "just 104 cal per serving," "for the whole family," "with naturally nutritious grains," "the healthy way to start your day." Any given box accompanies these tags with descriptive frames-such as "made from ingredients carefully selected from local farmers to meet the growing needs of our consumers, who appreciate wholeness through natural nutrition"- -as well as by a score of technical and commonsense concepts, such as "measuring," "calories," "micro-" and "macro- nutrients," "pleasure," "breakfast," "original breakfast," "healthy for you and your whole family."

The foodscape is overloaded with concepts like those camping on boxes of cornflakes. While some are frequently written down, others may be reserved to specific conversational contexts - as in the language of a sommelier or a butcher-or may even remain implicit_for example in cooking or eating practices. Jointly taken, these concepts play a crucial role in guiding our dietary behaviors, connecting them to a piece of medical advice, a lifestyle, a culture, or a tradition. More to the point, many of them also exemplify a poignant characteristic of food concepts that we wish to analyze-defectiveness.

In this paper we set out to offer a method of study for defective food concepts. These are, generally speaking, those concepts that fail to suitably represent a foodrelated domain or that offer representations that run counter to the interests of their users, taken individually or collectively. In employing the expression "defective concept" we line up with recent philosophical scholarship on so-called conceptual engineering, ${ }^{2}$ which makes ample use of it. At the same time, our work is also robustly grounded into different loci of the literature related to food, which have already discussed defective concepts, without giving them such a name. The concepts featured on boxes of cornflakes are a case in point of defectiveness: they typically bootstrap the meaning of the terms they employ, arousing the suspicion of promising more than what they actually deliver.

Defective food concepts demand up to date and targeted processes of rethinking or negotiation, which involve different stakeholders within the food discourse, and which need to account for reasons that are both contextual and principled. Philosophers can play an important role in such processes. In particular, and building upon the work done by Borghini et al. (2021) with respect to local food, we show

\footnotetext{
1 The cited disclaimers are taken from cornflake boxes currently sold on the marketplace, whose brand names we are omitting.

2 The Introduction to Burgess et al. (2020, pp. 1-25) offers a review of different uses of "defective concept," from Nietzsche to recent social and feminist metaphysics. Another extensive review is in Koch (2020), where the expression is characterized mainly with reference to the (in)capacity of a concept to represent an intended domain of discourse, either by omitting some of its elements or by failing to suitably represent them. Defectiveness is invoked also in Haslanger (2012), Thomasson (2017), Cappelen (2018), Manne (2018), among others. In the latter texts, we also find the idea that a concept is defective when it has undesirable effects on some (human or non-human) individual or collectivity.
} 
in the sequel that philosophers can offer means to represent chief disputes regarding food. Our study extends an approach to philosophizing that is not new (see e.g., Carnap, 1950) and that, in recent years, has been increasingly fine-tuned and put to use under the labels of "conceptual ethics," "conceptual engineering" (Burgess \& Plunkett, 2013), "ameliorative analysis" (Haslanger, 2012), and "conceptual negotiation" (Thomasson, 2017). As for food scholars, while they tend not to explicitly utilize "defectiveness" for referring to those concepts which they think should be revised, they offer numerous case studies of concepts that seem flawed and in need of revision. For instance, consider the pivotal work by Adams (1990) on the concept of "meat": after unveiling its underlying hierarchical, racist, and patriarchal significance, she proposes a revised understanding of it.

We begin (Sect. 2) by sorting out four dimensions that are key to assess the defectiveness of a food concept: (i) the data upon which it rests and the methodology by which those data are gathered; (ii) the ontological categories that it represents; (iii) the social acts that serve to negotiate and establish the concept; (iv) and the objectives and values that the concept is meant to reach. We then (Sect. 3) point out four types of defects that a food concept can have-fragility, polarization, incoherence, and schizophrenia-by examining two specific examples: local food and healthy food. Finally (Sect. 4), we close by suggesting the creation of situated conceptual maps as accessible tools for negotiation.

\section{Four dimensions of food concepts}

In our analysis, we shall rely on a minimal view of food concepts, which treats them as representational devices for states of affairs involving food in meaningful ways (Burgess \& Plunkett, 2013; Burgess et al., 2020). ${ }^{3}$ According to the view, also, any food concept is linked to a wide and varied network of representations. Consider, for instance, the concept "healthy food" and imagine the following four contexts in which it can be put at use: a lab where specific antioxidant properties of fruits are studied and tested; a dietitian chairing a weight loss group support; a blogger describing their morning routine to their readers; and an indigenous granny preparing a traditional meal for the grandchildren. These contexts will link "healthy food" to possibly overlapping, yet different, clusters of specific ingredients, recipes, dietary patterns, culinary traditions, lifestyles, medical conditions, brands, and so on. Therefore, whether "healthy food" manages to serve a valuable or intended role should be assessed in relation to a given context of agency and use. The need for contextualization-we submit-holds true to a greater or lesser extent for all food concepts. For these reasons, it is of utter theoretical importance to devise some tools for suitably capturing the contextual salience of food concepts.

With this in mind, we propose to frame our analysis of food concepts in terms of four dimensions. (1) Data and methodologies, which establish the empirical validity

\footnotetext{
3 Another traditional question on concepts is whether they are mental representations or abstract objects. We remain neutral on that controversy.
} 
of a food concept. Thus, in a given context of agency, we may primarily rely on empirical data-from microbiological to evolutionary, from nutritional to metabolic, gathered following science-specific methodologies. (2) Ontological categories, through which we impose certain structures onto our experiences, as when we regard a certain food (e.g., a croissant with a cappuccino) as a breakfast item. Such categories are not arbitrarily introduced nor are they part of the structure of reality: they rather emerge from the interplay between reality, culture, cognition, and semantics. (3) Fiat acts, which ground the existence of the concept in specific actors, such as the decision of a committee of experts to regard a certain food as a threat for a certain people. (4) Aims and values, as food is connected to identity, emotions, life plans, heritage, and many other value-laden domains of life.

These four dimensions are suggested by the different sorts of propositions that govern our understanding of food: propositions that rest on data and empirical observations; propositions that depend upon ontological categories imposed by cognitive and semantic constraints; propositions that rest on speech acts that seem to "make things with words" in a way that is peculiar; and propositions that depend upon value judgments. And yet, we recognize that the issues at stake emerge from a complexity that can hardly be resolved by philosophical analysis alone. As Parasecoli (2019, pp. 12ff) points out, in fact, a comprehensive understanding of food requires "knowledge in fields ranging from economics to politics, from agriculture to technology," which should involve a variety of different agents, each with its own expertise and values, such as "individuals and communities of consumers and producers, activists, distributors, marketers, retailers, chefs, scientists and nutritionists, designers and engineers, financial institutions, transnational corporations, local and national authorities."

In the remainder of this section, we offer a characterization of each dimension. Given the premises, we acknowledge that our analysis may not be all-encompassing; but, we believe it serves the much-needed theoretical purpose of introducing a conceptual frame to be employed by the broad community of food scholars and by the actors negotiating the use of key food terms.

\subsection{Data and methodologies}

Which data should ground a given food concept and which methodology should be employed to gather the data has been a hot and highly controversial topic. Two chief methodological camps can be sorted out here, a quantitative and a qualitative one.

The quantitative approach aims to measure and calculate the basic components of food and their function for sustaining human life on the basis of a given standard, i.e., nutritional needs and the qualities of food which should meet those needs. For instance, the number of calories, the presence of micro- and macro- nutrients, vitamins, and so on (Mudry, 2009; McClements et al., 2011; Scrinis, 2013). According to Carpenter (2003a) this approach traces all the way back to the chemical revolution that occurred at the end of the eighteenth century. It focuses on the relation 
between the physiology of the human body and some blocks taken as founding units of measure. ${ }^{4}$

The qualitative approach centers on the cultural, moral, social, aesthetical and structural role of food. The data are collected by surveys among eaters (e.g., Spence, 2017), sociological and anthropological analysis (e.g., Counihan \& Van Esterik, 2013), self-reflection of the human relations to food (e.g., Pollan, 2008), and structural studies on society (e.g., Biltekoff, 2013).

Both approaches can be cashed out in terms of their being more or less food-specific or consumption-specific - that is, whether their focus is on the eaten or on the eater. For instance, a quantitative approach can focus on, say, the nutrients found in a food item or in the digestive capacity of human beings, while a qualitative approach can focus on the relations between nutrients and ideology (Scrinis, 2013), or on the individual justification of a chosen diet (Pollan, 2008).

The number of data taken into account can vary considerably. In a simple network, each food concept is paired with just one kind of data, e.g., when nutrients are the only determinant of a healthy food. In a reductionist framework, the explanatory role of some data is reduced to the explanatory role of some more basic data, e.g., when nutritional data are reduced to those about the molecular composition of food (Ströhle \& Döring, 2010), or when a collection of eating habits is reduced to data about the economic relations that sustain them. In a more complex, multi-layered network, instead, food concepts are explained by different sets of data, e.g., food security (roughly speaking, the possibility of access to nutrient food) roots out on data regarding nutritional intake, economic, social, and environmental conditions.

Data gathering is surrounded by controversies. A chief one concerns their neutrality. As Biltekoff et al. (2014) efficiently sum up, purely quantitative data gathering tends to be presented as neutral with regard to other non-quantitative food externalities, including habits, conventions, beliefs, rituals, and taste. Yet, scholars demonstrated several hidden assumptions that underscore quantitative units of measure. These include: politics of the body and endorsed ideologies (Mudry, 2009); implicit biases of scientific research (Nestle, 2013); the dubious status of the basic components of the concepts that should be collected, e.g., whether nutrients or whole foods are to be measured (Jacobs \& Tapsell, 2007); which specific population is taken into account for collecting data (Pogge, 2016).

Conceptual negotiation could be employed to address issues surrounding data gathering, including their neutrality. In this paper, however, we shall focus on higher-levels of conceptual disagreement, which have so far escaped the attention of scholars and that involve negotiations concerning data and methods as a sub-part.

\footnotetext{
4 The specific units of measure have of course been shifting with time, based on scientific and cultural paradigms (Pollan, 2008; Carpenter, 2003a; b; c; d; Kamminga and Cunningham, 1995).
} 


\subsection{Ontological categories}

Food concepts represent a variety of different ontologies populated by a variety of categories: physical objects (e.g., dishes, meals), artifacts (e.g., recipes, ${ }^{5}$ restaurants), events (e.g., cooking, farming), conditions (e.g., hunger, appetite), processes (e.g., metabolism, foraging), perceptual states (e.g., bitter food, excellent food). ${ }^{6}$ How to comprise in one ontology all these different categories is the first complexity that food concepts should tackle.

A further kind of complexity derives from the different ontological commitments that agents who use the same concept may have. For instance, while to a certain agent the concept of natural food is nothing above a façon de parler, for another it is a concept tracking real properties of entities that carve nature at its joints. ${ }^{7}$

Mapping the heterogeneity of ontologies and ontological commitments could be conceived per se as a work of conceptual negotiation since an inventory of the ontological inventories - following the renowned Quinean metaphor-may be a useful tool for philosophers who want to spell out what are the ontologies of food concepts. However, also here, our priority is to tackle higher-level processes of negotiation, which would embed ontological issues as a sub-part.

For the purposes of the present discussion, we now provide a mapping tool for framing food ontologies based on a two-dimensional framework, visually consisting of two axes, each representing one dichotomy, and four quadrants where the different ontologies can be placed (see Fig. 1).

The first dimension of the framework represents the families of the domains of discourse where an ontology originates. That is, it shows an opposition between entities, which are posited by different agents in different domains, e.g., the domain of common sense and the domain of science. The dimension clusters, at one pole, all the ontologies whose entities rest on folk and common sense. Generally speaking, those ontologies are not the result of experimental methods but are the outcomes of more or less shared beliefs, based on first-person or collective experience. At the other pole stand ontologies populated by entities liable to experimental treatment or entities that are the outcomes, or the assumptions, of experiments, i.e., the so-called theoretical entities.

The second dimension stands for the approaches taken by the users who are meant to buy into those ontologies, i.e., whether they believe that the existence of the entities is grounded on how the world is or they are constructed by social and linguistic practices. The dimension clusters, at one pole, naturalist and realist approaches to ontology, which endorse the existence of the posited entities regardless of conventions, beliefs, languages, and conceptual schemas. On the other pole, we group

\footnotetext{
5 The ontology and the philosophy of recipes have been the center of a recent interest by philosophers, see Borghini (2015, 2022), Borghini \& Engisch (2022).

${ }^{6}$ For some recent developments of formal food ontologies, see Dooley et al. (2018); for some basic principles for constructing food ontologies in the light of their future, see Borghini et al. (2020a).

7 More realist positions entail that ontologies are independent from agents, however we are here framing the debate and not taking a stance over that. Hence, we employ a language as commitment-free as possible just to stress the differences among positions.
} 
constructivist and relativist approaches, which claim that the existence of those ontologies depends on conventions, beliefs, languages, and conceptual schemas. ${ }^{8}$

For instance, a diet based on nutritional intake typically rests on nutritional science. The entities posited (i.e., nutrients) crucially depend on the experimental method, which is also used to link food components with human metabolism. This ontology is artlessly undertaken with a realistic or naturalistic stance and finds its place at quadrant 3. By contrast, consider a classic approach of structuralist anthropologists, that sees food as a socially constructed entity to the extent that anything can turn into food, if a community believes that it is such. This ontology would be placed in quadrant 2, since its domain is delivered by common sense and it is taken as constructed or relative to some situated beliefs.

The two-dimensional framework can be used for clarifying the "distance" among two stakeholders in negotiating a food concept, facilitating the individuation of specific disagreements and focusing the discussion. It can also serve as a tool to fine-tune the ontological nuances of complex concepts, such as geographical indications, ${ }^{9}$ where scientific knowledge and common sense, realist and constructivist approaches, can often be mingled together in the same document or discourse.

\subsection{Fiat acts}

Data and ontologies are linked to concepts by means of specific acts on the part of specific agents. Following Smith (2001), we call those fiat acts. They can be characterized based on two aspects. The first is the type of agential authority, while the second is the kind of knowledge that guides the act.

Authority. A fiat act can be based on an absolute or relative form of authority. Each of them, in turn, may be performed by an individual or a community. ${ }^{10}$ When absolute, the agent - be it an individual or a community — sets the boundaries of a food concept regardless of other networks of agents. For instance, the fact that a True Neapolitan Pizza should be consumed within 10 min of production as dictated by The Neapolitan Pizza Association is an example of an absolute fiat act exerted by a community of agents.

A relative fiat act, instead, rests on a source of legitimacy which does not depend on the involved agents. An example of an individual relative fiat act is given by an expert who owes their authority to an institution, e.g., a biologist who should decide what microbes can be safely included in a given fermentation process whose general guidelines are established by a sanitary bureau. A community-relative fiat act is when the authority of a group is conferred by external authorities, e.g., whether a

\footnotetext{
${ }^{8}$ For a classification and an analysis of the different realist and anti-realist positions on food, see Borghini \& Piras (2021).

9 Labels that link the identity of a food product to a specific place of production, which would allegedly bestow inimitable and distinctive properties to the product. For further discussion of this topic, see Sect. 2.1.

${ }^{10}$ We are well aware that an act exerted by a community may differ from an act exerted by an individual in many ways. However, addressing it goes far beyond the aim of the paper. For a more in-depth discussion on the difference between groups and individuals, see Tollefsen (2015).
} 


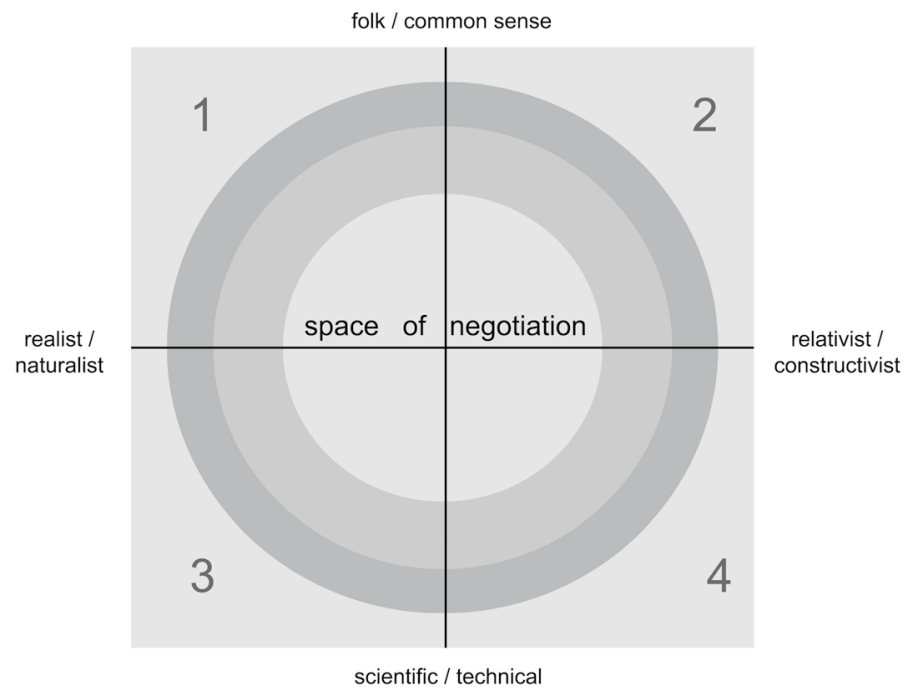

Fig. 1 Space of negotiation between different ontologies

wine is a geographical indication is due to a cartel of producers who set up the characteristics that should be met by the wine. However, their authority should in turn be officially recognized by national and supranational agencies, e.g., the European Union. $^{11}$

Knowledge. Fiat acts may require specific knowledge to be performed. When it comes to food, the literature (Borghini et al., 2020b) spotlights three types of knowledge. (i) Scientific and technical knowledge. Fiat acts concerning certain food concepts should involve objective and measurable data, or the ability to master specific technical instruments. This is the case, for instance, of basic food categories such as milk, cheese, or tomato, whose understanding may arguably depend upon the evidence produced by specific scientists; or the concept of safe food, which depends upon the knowledge of technical experts. (ii) Traditional ecological knowledge (TEK). This is a broad category that comprises all the ways of sorting out concepts based on "local" (i.e., contextually specific) clusters of expertise, such as those arising from local agricultural and breeding practices, and traditional beliefs. Certain salmon taxonomies, for instance, require that the assignment of a fish to a taxon be performed by a local salmon expert (Lien, 2015). (iii) Practical knowledge. Fiat acts founding certain foods depend on knowledge established through practices, interests, contextual and non-systematic goals that cannot be rendered in a principled way and that, at the same time, do not constitute forms of TEK. Instances are practices of recipe making, such as baking bread, which may depend upon contextual elements and specific know-how.

\footnotetext{
11 For a classification of types of recipes based on the authority of their authors, see Borghini \& Gandolini, (2020) and Borghini (2022).
} 
The variety of fiat acts that can set the boundaries of a food concept is conducive to a host of disagreements and confusions. Take, for instance, Parmesan cheese. Who has the authority to establish whether a certain piece of cheese is or is not Parmesan? Is such authority granted in virtue of some specific knowledge or in virtue of some other principle? Again, we shall not engage in conceptual negotiation at this level (see Borghini, 2015, 2022; Borghini \& Gandolini, 2020), but rather we consider fiat acts as one of the sub-components of a more comprehensive conceptual negotiation about food concepts.

\subsection{Aims and values}

Food concepts may be upheld for different aims and values and philosophers are naturally in a good position to assess and negotiate them. In practice, it is often impossible to disentangle them. But, from a theoretical standpoint, they can be divided into two broad categories, namely epistemic and social aims or values (Brigandt \& Rosario, 2020). Let us see each of them.

The epistemic aims and values of a food concept relate to its degree of accuracy and contextual relevance. These aims can be either terminal, i.e., the concept should produce accurate and contextually relevant beliefs about a food domain, or instrumental, i.e., the concept should produce accurate and contextually relevant beliefs about other concepts (or they could be employed as instruments for producing such beliefs). For instance, one of the epistemic aims of a certain interpretation of local food is to certify that the foods that satisfy the application conditions of the concept have been produced in a certain region (e.g., Adams, 2018). The concept of food miles, i.e., the distance between the production and the consumption places of a given food measured in miles, is instrumental to the concept of local food since it provides the metrics for certifying whether a food is local or not.

The social aims and values of a food concept relate to its role in bringing about desirable social conditions (Haslanger, 2012), which may be explicitly (i.e., directly) included within the concept, or only implicitly (i.e., indirectly). For instance, preventing famine is a direct aim of food security (FAO, 2006), while it is an indirect effect of food sovereignty that enabling local farmers to govern food chains could also allow them a free, stable, and continuous access to food.

Arguably, a good concept can reach its aims or express and embody a value. If it fails, two strategies can be deployed-resetting the concept or doing away with it. For instance, the concept of nutritious food has been reset several times throughout the history of nutritional science to better reach its aim, namely being adequate for human dietary intake (e.g., Scrinis, 2013 offers a significant overview, from the quantification of the components of a food to the study of its specific functions with respect to a specific human need).

However, some concepts may not be suitable to be reset, for instance because they are representationally inadequate or because they have become practically useless or even harmful. An instance of the first case may be the UNESCO concept of the Mediterranean Diet, which, according to several scholars, fails to represent the variety as well as the social structures and historical patterns of the many diets 
widespread in the Mediterranean regions (Simopoulos, 2001; Simopoulos et al., 2000). An instance of the second case is the famous 1992 USDA's food pyramid (and its successors), which flunks its target of instituting a balanced and scientifically sound diet for the population at large, therefore failing to adequately counter health issues related to food consumption (see Nestle, 2013, pp. 51-67; Mudry, 2009, pp. 77-136).

Lastly, a food concept may also hold wrong aims or bad values, both on epistemic and social grounds. For instance, Scrinis (2013) claims that the concept of functional food, which reduces food to its capacity of improving human bodies, cannot give us a comprehensive and genuine knowledge of food. At the same time, functional food promotes the wrong aim of making the body more suitable for commodification as long as it reduces nutrition to the goal of improving bodily functions for increasing the profit of private companies. Thus, he contends, the concept should be dismissed.

\section{Defective food concepts}

The four dimensions of food concepts outlined in the previous section provide one of the two main sides of our analysis. We shall now turn to the other side, namely the avenues that render a concept defective. As we already noted above, the literature suggests that a concept may be defective either by failing to represent its intended content or by fostering undesirable social consequences. Below we spell out these two avenues in some details. Our analysis will be specific to the food domain, though what we outline may hold for a wider range of inquiries that avail themselves of defective concepts.

Failures in representation obtain when a food concept fails to suitably represent an intended food domain by excluding or misrepresenting some or all of its dimensions, namely the intended data and methodologies, the ontological categories, the fiat acts, and the aims and values. For instance, the concept of "novoandino cuisine," as forged by Peruvian starred chefs, is charged with misrepresenting meals and dietary patterns of Peruvian rural communities who are deprived of a fair representation of their cultural heritage (see García, 2013, 2021). This failure may be explained by pointing out that (the data and methodology, ontological categories, fiat acts, and aims and values of) "novoandino cuisine" represent a counterfeit version of Peruvian gastronomic culture designed to earn a profit for the few starred chefs in Lima. Another notable example is offered by fair-trades labels, which have been criticized for smuggling unethical products under the "fair-trade food" tag (see Sylla, 2014, pp. 34-58). In this case, the failure can be explained by stressing the shallowness of certain certifications (that take into account only one phase of the complex processes of production and distribution), the polarization of different fairtrade labels, and the incapacity of many of those labels of reflecting fair aims.

Failures in the social consequences obtain when the food concept has undesirable consequences for some or all of its stakeholders. For instance, as Adams (1990) argued, the patriarchal understanding of "meat," by placing meat as the main dish, mirrors and reinforces unjust and abusive hierarchical relationships as well as the 
social exclusion of women and non-Western people. In this case, the concept "meat" fails to integrate stakeholders' goals: it aims at covering all the ways of eating, while its first three conceptual dimensions are filled up by objects, patterns, and relations privileging meat consumption. ${ }^{12}$

The two avenues for failure are interwoven, as undesired social consequences are often traced back to the fact that a concept fails to fairly represent a domain. Even when there is no overlap, defects may be distinct and yet comparable in some respects. It may in fact be feasible to compare conceptual failures in the number of defective dimensions (we have, thus, mono-, bi-, tri-, and four-dimensionally defective food concepts). For instance, if the aim of a diet is to prevent animal suffering, then a diet with a lower presence of food of animal origin is less defective than a diet including a higher level of animal food. At the same time, comparative assessments of defectiveness risk incurring significant margins of error and we shall not back up their generic usage here. This is because it is often difficult to find an evaluative standard agreed upon by different stakeholders and shared across different contexts. For instance, while it may be crucial for legal and economic purposes to pin down whether a plant-based hamburger is meat, for the sake of ordinary conversation the question may not arise at all or come off as brainy and futile.

These two major avenues of defectiveness for food concepts, by themselves, are unable to explain where the defectiveness of a given food concept may lie, in a way that suggests how the concept may be amended, negotiated, and hopefully improved. A more promising strategy we wish to pursue here is to put together the two avenues with the analysis of the four dimensions we proposed in the previous section. By means of this strategy we can pinpoint why a given concept is defective, that is, which (aspects of the) dimensions of the concept make it defective.

Now, the dimensions of a concept can be linked in multifarious ways to the avenues of defectiveness. To provide some order to our analysis, we introduce four types of defects that may affect a food concept. These defects-we contend-cover the most common sources of conceptual failures when it comes to food, though of course they do not pretend to exhaust all the possible conceptual failures that have and will emerge in food cultures. We first briefly characterize the defects, to then exemplify them. As it goes, they typically emerge when different stakeholders buy into different and conflated versions of the same concept, without negotiating a common content and structure.

(a) Fragility, that occurs when a concept does not deliver definite or informative contents with respect to the four dimensions. A fragile concept may fail to include sufficient information for representing what it is supposed to refer to, or feasible indications for the aims it strives for.

\footnotetext{
${ }^{12}$ In addition, the concept is defective also in the first sense: it does not provide an exhaustive representation insofar as it conceals both animal sufferance and women's submission (in its data and ontology); also, it tends to exclude women from the fiat acts determining the boundaries of the concept of "meat."
} 
(b) Polarization, that occurs when the concept can stand a plurality of mutually inconsistent interpretations leading up to some clashes (e.g., over ethical, political, or cultural values).

(c) Incoherence, that occurs when there is a mismatch between the first three dimensions of the concept and its aims.

(d) Schizophrenia, that occurs when there is a combined endorsement of dimensions pertaining to different instantiations of a concept. A schizophrenic concept represents disparate non-mutually related states of affairs, resulting in being practically useless since it is unclear what are its contents and aims.

We shall now illustrate how the four defects actually affect food concepts. To do so, we chose two widely used examples-local food and healthy food. Before moving on to that task, however, one more issue shall be addressed, albeit briefly: whether the degree of defectiveness of a food concept is owed to its internal constitution or to external conditions.

It is tempting to think that some concepts are defective due to some internal conditions. Plausible candidates may be those concepts having a partisan origin and promoting unjust or unsound principles, like the already mentioned concepts of "Mediterranean Diet," "food pyramid," and "functional food," which have been charged with internal flaws. Also, a concept may be misappropriated by an agent or a group of agents for achieving consequences that are undesirable to another agent or group. A case in point is "extra virgin olive oil," which—at least according to Mueller (2011) — has been used for selling adulterated oil and hence defrauding consumers. $^{13}$

At the same time, some food concepts may become defective after serving desirable purposes because of changing external conditions. This is for instance the case of concepts that are no longer able to represent a food because the food itself changes, like the shift in underlying conditions that fixed the meaning of key food items such as "bread" and "yogurt" occurred in the US market in the 1970s (Pollan, 2008, pp. 148-154), or like the foods that had been contaminated with nuclear waste during the Chernobyl disaster, after which food concepts such as "fresh milk," "local berries," and "wild weeds" abruptly changed, from referring to safe and nutritious food to hazardous ones. Shifting social conditions may render a concept defective, too. A case in point is "wild food" when applied to non-Western foods, which can hardly explain different production and consumption patterns and, hence, turns into a fragile or schizophrenic concept (Borghini et al., 2020b).

The possibility that some food concepts are only externally-and not internally-defective suggests that not all food concepts are constitutively defective. For instance, the literature suggests that "organic," as originally conceived, was highly functional to its scopes, i.e., providing foods totally free of synthetic additives and

\footnotetext{
13 This may lead a reader to surmise that at least some internally defective food concepts are included in the ranks of so-called essentially contested concepts (Gallie, 1955), as suggested for instance by Thompson for "sustainability" (2010, p. 196). We thank an anonymous referee for bringing to our attention this point.
} 
pesticides (see Fromartz, 2006). Other examples are provided by scientific concepts that are not defective in an original context of application, e.g., the concept of ketogenic diet when applied in controlled conditions for healthy losing weight to severely obese patients.

\subsection{Local food}

"Local food" is at once a model for sustainable dieting, a tool for socially valuable interaction, and a precious label in the market (Borghini, 2014; Gray, 2014; Yeung, 2014). It is often maintained that foods locally produced fit many ethical claims about alternative food production, the preservation of cultural heritage, social cohesion, sustainability, and so on (see McWilliams, 2010 for a critical overview). Yet, defining when a food is local is not as easy as it may seem. Building upon the study of local food begun in Borghini et al. (2021), we sort out three takes on the concept, which differ widely across the four conceptual dimensions. In the sequel, we provide a characterization of each take on the concept and sum up our analysis in Table 1. Then, we use the table to illustrate the four types of defects that a food concept can be charged with.

$L F 1$. This is a distance-driven take on local food, according to which a food is local when it is consumed at less than some fixed distance to the production place. In this case, the data are the miles traveled by the food from the production place to the consumption one(s); the ontology is constituted by foods that have traveled less than the fixed distance; the fiat act is accomplished by an institution that fixes the number of miles and certifies how many miles a food has actually traveled; the aims and values typically involve the reduction of pollution due to food transportation, the improvement of local economies, or providing local consumers with fresh and safe food (e.g., Werkheiser \& Noll, 2014).

$L F 2$. This is a terroir-driven take, according to which a food is local when it owes its specific qualities to its place of production (i.e., climate, sun exposure, soil, rainfall) and to the traditional techniques rooted in that place, e.g., the Italian cheese Bitto owes its flavor to the environment where the cows that produce the milk grow and the curdling techniques adopted by local farmers. ${ }^{14}$ In this case, the data are provided by the qualities featured by the food and the qualities of the place, in a sense as broad as including local practices; its ontology is fixed by a twofold framework, which includes the historical relations of the food to its production place and the causal link between the place's properties and the food qualities; the fiat act is granted by an institution, e.g., a consortium, which establishes and certifies the link

\footnotetext{
14 This take is well explained by Montanari in his 2006. There, he says (p. 75) that: "all food is by definition "local," that is, of the region, especially if we think of popular culture, which is more directly tied to local resources. But even on a higher plane, even when the decisive variant of the marketplace enters into play, the attention devoted to products labeled as "of specific origin" is not an innovation. One could cite as examples an endless number of authors and leading figures to show to what extent awareness of terroir, of local conditions and resources, has always constituted an essential given of food culture."
} 


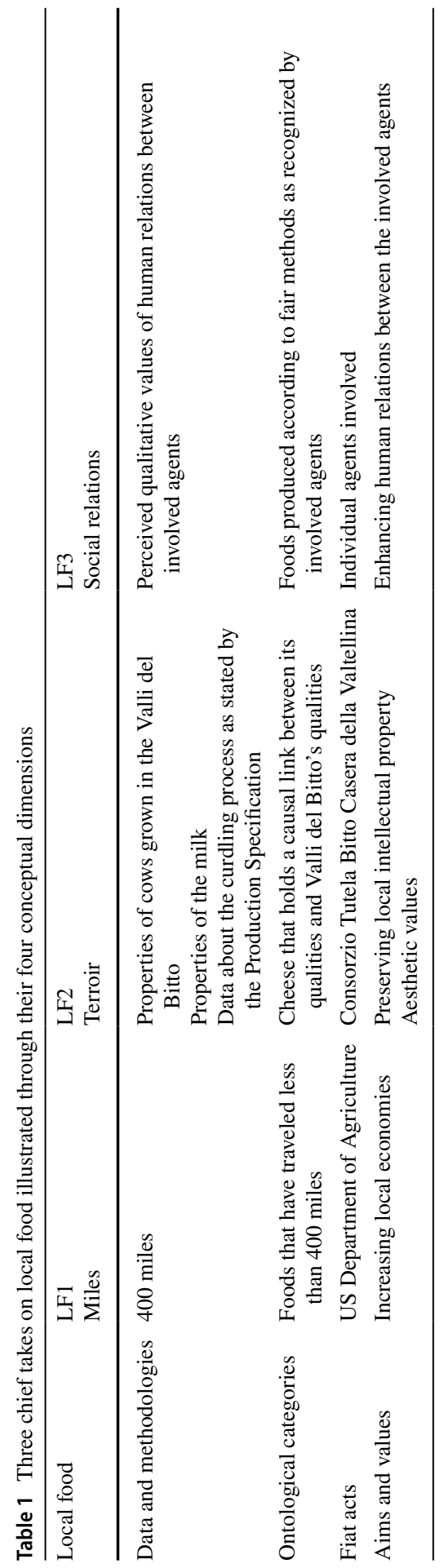


between the qualities of the food and the qualities of the place ${ }^{15}$; the main aim of the concept is to ground the intellectual property of the food to the producers located in that place (e.g., Giovannucci et al., 2009).

$L F 3$. This is a socially driven take, according to which a food is local when the social distance between the different stakeholders is as short as possible in order to promote a joint protest against developmentalist forms of production. The data are the qualitative relations between consumers and producers; the ontology is populated by food items produced through just labor conditions, such as fair wages and trustworthiness; the fiat acts are the agreements stipulated between those agents, which jointly decide whether a food respects the criteria for being local, i.e., whether it has been produced in a fair way; the aim is preserving human relations between agents involved in food production and consumption (see Ho, 2020).

Table 1 below sums up these three takes on local food via specific examples, showcasing the variability of the concept across its four dimensions. Each row stands for one of the four conceptual dimensions, while each column represents a different instantiation of the concept of local food. We selected as an instance of LF1 the general guidelines about local food provided by the US Department of Agriculture; LF2 instantiates the Protected Designation of Origin specification of the Bitto cheese, i.e., Consorzio Tutela Bitto Casera della Valtellina; as an instance of LF3 we picked the concept of cosmopolitan locavorism recently developed by some Hong Kong young farmers and activists (Ho, 2020).

The table organizes the different instantiations of local food by teasing out their dimensions. It serves also to highlight the four defects that can affect a food concept—fragility, polarization, incoherence, and schizophrenia. We now provide a few examples for each kind of defect. ${ }^{16}$

Fragility. Let us consider the data of LF1 and the methodologies adopted to gather them. The instantiation of the concept aims to increase the values of local economies, as established by the US Department of Agriculture, which also states that a food can boast of the title "local food" if it travels for no more than 400 miles. However, this distance is controversial: why the interests of local farmers can spread up to 400 miles? Isn't there the possibility that different local interests conflict? In fact, according to some who still endorse this view, the number of miles should be considerably shorter, i.e., 100 miles (Smith \& MacKinnon, 2007). As it would be easily imaginable, the distance cannot be fixed once for all since there is no objective or shared criterion of the measurement. This instantiation thus proves to be fragile inasmuch as it cannot provide a fixed method to establish how many miles a

\footnotetext{
15 See Holt and Amilien (2007, p. 5) who made this connection explicit by claiming that "European regulation is a common political tool that creates a logical frame for reflections about local food in Europe, and the most common reference in this special issue has been the EU regulation No 2081/92 from July 14th 1992 about Protected Designations of origin for foods, which is characterized by the name of a place to designate products coming originally from this area and therefore having a specific quality."

16 The aims and values here displayed serve only for the sake of exemplification of a more complex and diversified reality. In fact, it is still possible that each instantiation has multiple aims and values, or that it has none at all. Also, the table does not set forth an instantiation of the concept without an aim, which would be a paradigmatic case of fragility.
} 
food can travel while remaining local. LF3 might be fragile too, as long as its data crucially depend on the feelings of involved agents, such as social cohesion, which are surely difficult to measure.

Polarization. Although the different takes of the concept may apply to different contexts, they are frequently employed in the same one. In this case, takes may conflict with each other ending with the polarization of the positions. For instance, consider when an international organization, e.g., the European Union, decides what should count as local food: should it endorse LF1 or LF2? If it endorses LF1, then many geographical indications nowadays considered as local food would be ruled out, e.g., Cioccolato di Modica, a renowned chocolate from Sicily, whose cacao seeds come from Mexico, would no longer be considered a local food.18 While, if the European Union would endorse LF2, then a European producer, say, of bananas, could not claim that the bananas it produces are local. In fact, bananas do not hold any special link to Europe and thus they match neither the data, nor the ontology of LF2. As long as these two instantiations cannot be integrated, they turn out to be polarized within the same context, i.e., the legislative camp of the European Union.

Incoherence. The instantiations of the concept may often fail to reach their aims or express a value due to an incoherence between the contents of its dimensions. For instance, LF2 could fail to preserve local intellectual properties, if the place where a certain food is produced is owned by non-local companies that however fulfill all the other dimensions. Indeed, the dimensions of LF2 fail to specify who should own the specific place where the food is produced. In that case, the intellectual property of that food is not held by local producers. Thus, the aim of the concept is not accomplished, even if all the other dimensions are respectively satisfied.

Schizophrenia. Some actors, more or less consciously, may endorse the contents of dimensions pertaining to different instantiations of the same concepts, ending up mixing them in a concept that is not well-integrated. In fact, very often, it is not clear at all who can and should set up a concept and what is the normative standard they should abide by. Consumers' associations and cartels of producers provide different conflating notions of local food feeding a schizophrenic understanding of it. In fact, the absence of a common negotiation and a shared evaluative standard may result in allowing the most diverse versions of a food concept. For instance, a survey by Durham et al. (2009) shows that many American consumers endorse LF1. However, they replace the food miles, i.e., the data of LF1, with the State where the food was produced. Thus, a food produced in Texas is local when it is consumed in Texas, regardless of whether the distance between the production and the consumption places exceeds 400 miles. It is clear that local food could no longer be based on an ontology that rests on miles, but might instead switch to, say, the ontology of LF2. Local consumers can indeed justify their belief that the food produced in their State is comparatively better than the ones produced away due to a special link with its place. This new take of local food is composed of a new datum and the ontology of LF2. The fiat act can in turn be charged by the involved agents, who should recognize where the food comes from. It somehow matches the fiat act of LF3. Its aims, finally, may remain the ones of LF1.

The resulting take appears to be schizophrenic for a number of reasons: its data and its ontology mismatch: the State where a food comes from, which is the new 
datum of the take, does not suffice as a basis of an LF2 ontology since it does not spell out the relations between the qualities of a food and the qualities of the place; the fiat act so redefined may, in turn, fail to provide a basis for the ontology of the take. The agents involved, who basically are the consumers, could not have the right expertise to justify the relation between a food and a place. Finally, the aims of LF1 are disconnected from the other dimensions. The same State may be populated by different competing local economies, so the new datum can hinder that aim; the link between food and place, as required by ontology, can undermine the aim of LF1 since a local farmer may also decide to produce more convenient food that is nonetheless not historically rooted in that place of production. Therefore, due to the lack of internal integration, this take on local food fails to accomplish its intended aim.

\subsection{Healthy food}

Healthy food can be framed either in terms of the absence of harm, i.e., the idea that food must not be poisonous, or in terms of benefits, i.e., food should enhance health (Murphy, 2020). As many scholars suggest (e.g., Siipi, 2013), it is disputed whether healthy food is a concept that should consider a single food item as capable of being healthy for us, or it should consider the healthiness of a whole diet, or of ways of consumption. It turns out that what healthy food means is unclear. We propose four different instantiations of the concept of healthy food to be broken down in the comparative table.

HF1. The first is nutritionism (Scrinis, 2008, 2013), which claims that healthy food has a specific configuration of nutrients. It identifies a reductionist paradigm characterized by a microscopic focus on food composition: food-body-health interaction is understood and framed in biochemical terms. Nutritionism is the dominant ideology within nutrition science, and implies a reductive approach to health as well: as Scrinis puts it, health is reduced to a set of diet-related and quantifiable biomarkers, indicators, and functions. A typical expression of such reductionism is the association of a bodily process to particular food components and the assumption that health can be assessed through that specific association (e.g., glycemic index, body mass index). Data comes from the biochemistry of food and the frameworks of biomarkers; its ontology is populated by nutrients and their relations with human bodies; the fiat acts are performed by nutrition scientists, corporations, and individuals, when they endorse the nutritionist gaze (Scrinis, 2008); the aim is twofold: reaching health via nutritionally balanced diet; aligning individual food choices to the structures of the dominant agrifood system by shaping bodily functions ways that are manageable for science and institution, profitable for the food industry (Biltekoff et al., 2014; Hayes-Conroy et al., 2014; Scrinis, 2008).

$H F 2$. The second instantiation invokes the idea that food is healthy when it is natural. This association has been well investigated by Siipi (2013), who concludes that there are multiple meanings of naturalness connected to the healthiness of food, e.g., "natural" as "familiar," "natural" as "authentic," "natural" as "what satisfies needs," "natural" as "lacking human influence" (Siipi, 2008). The equation between healthy food and natural food never comes with an explicit reference to the kind 
of un/naturalness involved, making this conception of healthy food particularly problematic at its internal level. Both data and ontology are indeed dependent on the kind of naturalness taken into consideration: for "natural" as "customary," for instance, cultural norms would be the data, and the food ontology will be ethnocentric (e.g., insects would not count as healthy food for individuals of a certain culture, because they are considered unnatural/not familiar).

HF3. The third instantiation centers around the principles of ecological nutrition, according to which the healthiness of a food depends upon the entire socioecological life of the item, from production to digestion. Data comes from the ecological system, food system, and human body (Klein, 2013; Guthman et al., 2014). The approach is intrinsically interdisciplinary: methodology from ecology, biomedicine, and agricultural sciences are applied to produce a systemic and integrated knowledge of food. The ontology is relational: different ontological layers are taken together, from the fine-grained entities to bigger biocultural networks. Fiat acts come from scientists, activists (take for instance the HFHC movement) ${ }^{17}$ and institutions, but also from customers looking for more and complex information on the life-cycle of food they are purchasing. The aim of this approach is to consider food healthiness in the light of an ecological perspective, in which human bodies are systems connected to the broad environment: the health-related costs and benefits of food should be then assessed through this wider ecological network.

HF4. The fourth instantiation is the nutritional epigenetic approach: the idea that what is healthy is defined by our genetic response to dietary inputs. Like HF3, this approach endorses an increasingly relational biology (Landecker, 2011), more focused on network relations than on internal function and structures of single living entities (Powell \& Dupré, 2009). Both HF3 and HF4 are sensitive to an ontological shift in understanding biological organization; in the definition of the environment (human body and food can be framed as environments at the same time) and metabolism, as a zone of regulation rather than energy conversion (Dupré \& O'Malley, 2009; Landecker, 2013a, b). The data are the effects of nutrition on patterns of gene expression/regulation, gathered for instance through experiments on mice microbiota; fiat acts are performed mainly by researchers in areas like gene ecology, medical microbiology, epigenetics, system biology, gut-microbiome sciences and so on. This instantiation of healthy food goes in the direction of a stronger interplay of the nutritional and therapeutic functions of food, to the point that dietary inputs can be conceived as preventive and care tools.

By means of Table 2, we can illustrate the four conceptual defects that may affect a food concept.

Fragility. Among the four instantiations of the concepts of healthy food, HF2 appears as the most fragile. As mentioned above, the diversity of meanings that could be attributed to "naturalness" affects the concept of healthy food through

\footnotetext{
17 The Healthy Food in Health Care is a movement that advocates for a redefinition of healthy food within healthcare institutions. As Klein (2013, p. 2). puts it "the body and its disease or well-being are at the center of serious contestations over the food system, right use of the landscape, and appropriate use of agrifood technologies."
} 


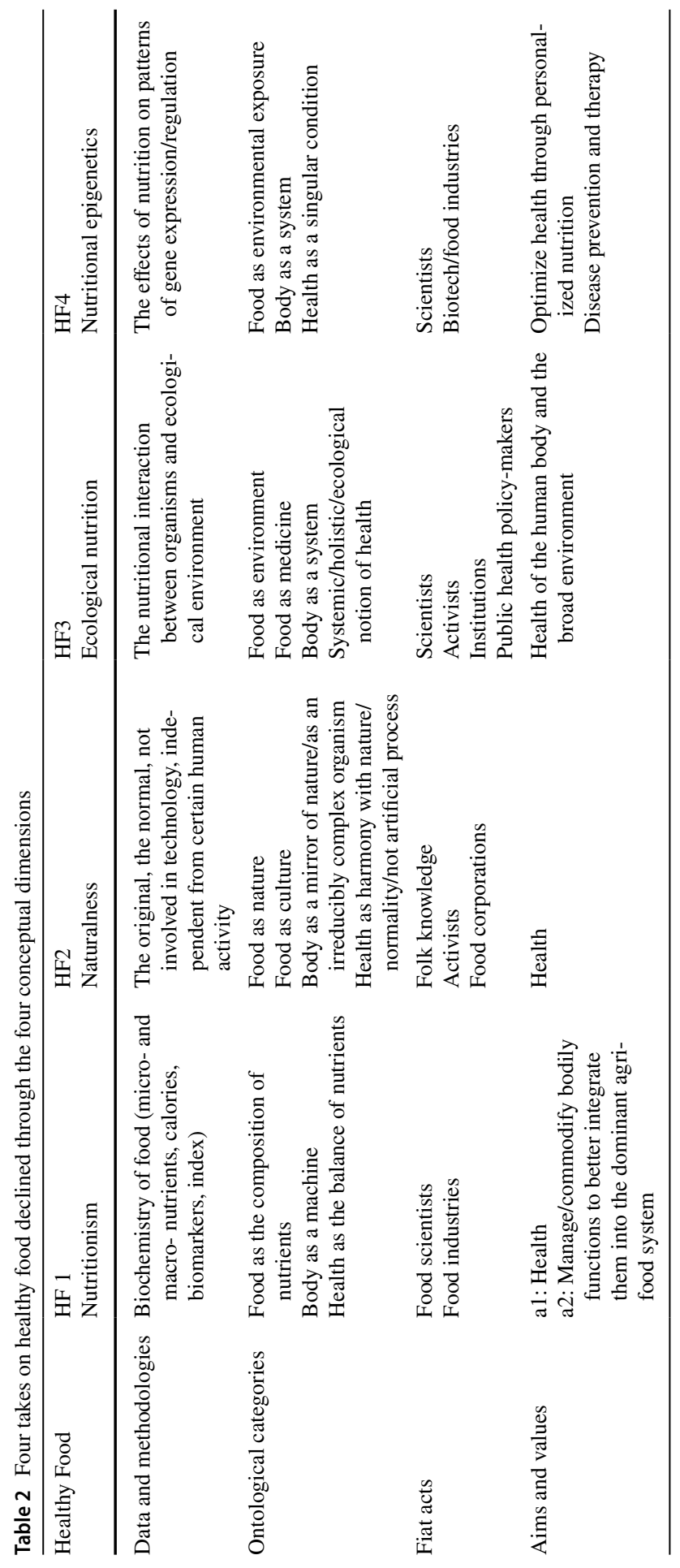


all the dimensions - to the point that a comparative table for the family of HF2 could be easily imagined. A potential element of fragility can be found in HF4, in the data and methodologies dimension: many different disciplines converge under the label of nutritional ecology, with their own methods, taxa, and concepts. Such interdisciplinarity is a resource only when a methodological integrated framework is at play (Raubenheimer et al., 2009). Furthermore, each of the four instantiations tends to underestimate the role that unhealthy eating plays in relevant social occurrences, both formal and informal. Unhealthy foods may in fact enable experiences that provide highly desirable values, such as family or community identity, crucial for human psychological health (e.g., celebrations with specific cakes, see Barnhill et al., 2014). In this respect, each of the four instantiations could be marked with fragility since they do not include in their ontology specific food entities relevant for human well-being and they do not consider the aim of health in its multifactorial composition. $^{18}$

Polarization. Both HF1 and HF4 focus on the fine-grained layers of molecular interaction. The idea that diet affects physiology is at the very core of both positions but in substantially different ways. HF1 promotes the calculus of input-output, in which the body is the machine where transformation happens, and food is the measured input associated with projections on our body image and health - eat fewer calories to lose weight, take more vitamins to contrast aging. HF4 is not about knowing food components to create the right equation within the body-machine, but rather about how food affects our phenotype through mechanisms of attachment and removal of chemical marks to our DNA. In other words, it is about how food rules and shapes the metabolic equation (Landecker, 2011, 2013b). Two polarized concepts of metabolism and the human body are entailed in HF1 and HF4: clinical research targeting nutrition-related disease, for instance, would likely encounter some obstacles if both concepts are at play simultaneously within the research teams. As shown in Sect. 1.1, another kind of polarization occurs between HF1 and, for instance, HF3.

Incoherence. HF1 aims were divided into two: (a1) is the overt aim; (a2) refers to the broader function of nutritionism, that is, adapting our knowledge and practices about food and the body to the requirements of the agrifood industry (Scrinis, 2008). Nutritionism, thanks to its reductionist account of food, frames human physiology in manageable terms, facilitating its alignment with the dominant ways in which food is produced, processed, retailed, marketed, and consumed nowadays. The effectiveness of nutritionism for health has been largely disputed, both by food scholars and food scientists, mainly due to the oversimplification of eating and diets; the standardization of individual traits; the promotion of ultra-processed food through their supposed health and functional benefits (Pollan, 2008). Accordingly, we would consider HF1 coherent with a2, but incoherent with a1.

Schizophrenia. Supermarket shelves, with packaged and labeled food on display, overflow with examples of schizophrenic healthy food concepts. The plethora of tags and descriptions are, indeed, examples of how we tend to encounter the concept

$\overline{18}$ We thank an anonymous referee for bringing this point to our attention. 
of healthy food: dimensions pertaining to different instantiations of the concept are combined, and endorsed simultaneously by a product. Take for instance a granola bar marketed as "healthy." Imagine that " $100 \%$ natural grains" is written down at the center of the green-washed packaging (HF2); at the same time a tag, slightly shifted on the left, stresses the bar's biochemical features, e.g., "low sodium" (HF1); "no genetically engineered ingredients" shows up on the package as well, entailing a reference to the ecological history of the product (HF3). The bar endorses at least three different concepts of healthy food and the aim is not clear: what kind of health is fostered here? The tags may refer to different kinds of food values (e.g., naturalness as good per se) as well as they may jointly underlie a comprehensive notion of healthy food which includes all the aforementioned takes. ${ }^{19}$ However, it may also be possible that the tags refer to different mutually inconsistent takes' aims: having low sodium may be inconsistent with, say, the aim of being made by $100 \%$ natural grains. In fact, natural grain can be modified at a later stage for matching the lowsodium goal. This, in turn, is in stark contrast with HF3, while may be consistent with HF4.

Following Scrinis (2008), we could argue that creating conceptual confusion towards the healthiness of food is a commonly adopted marketing strategy, to which then, the same food industry responds by offering a simplified (HF2) and reductionist account of food (HF1) to anxious consumers. It seems therefore that some instantiations of healthy food show greater adaptability to, or even benefit from, situations of conceptual confusion.

\section{Open problems}

Our goal in this paper was to propose an analysis of the structure of food concepts and of their defects. We highlighted four dimensions for these concepts and used them to expose four types of defects. Providing methods to address these defects is an open problem that requires far more space than what we have here and that we reserve for the future. Still, what we have done so far clearly indicates plausible developments of our view.

First of all, our analysis brings to light three key areas of conceptual negotiation for food concepts. The highest level of negotiation takes place between different instantiations of a given concept, e.g., different takes on local food. At an intermediate level, we negotiate between different dimensions of a single instantiation, e.g., about which fiat acts should ground a distance-driven take on local food. At the lowest level, the negotiation concerns one specific dimension, and we pointed out several examples of this sort of negotiation along the paper. The two case studies we discussed-local food and healthy food-well illustrate the three areas of conceptual negotiation.

19 We thank an anonymous referee for pointing out this. 
Secondly, as the case studies we survey demonstrate, our approach can be put to use in providing a conceptual map of a given food concept, which would be divided according to the three levels and would highlight critical spaces of negotiation.

The work ahead of us, then, is to further clarify the three levels of negotiation and how they can be employed to develop conceptual maps for negotiating food concepts. Conceptual maps seem a promising tool for addressing the challenge of integrating the vast and varied knowledge about food that scholars and practitioners have impressively recorded in recent years. In this sense, our study stands as an example of how philosophers can serve the much-needed role of bridging across different camps of knowledge, also when it comes to food and eating. ${ }^{20}$

Funding Open access funding provided by Università degli Studi di Milano within the CRUI-CARE Agreement.

Open Access This article is licensed under a Creative Commons Attribution 4.0 International License, which permits use, sharing, adaptation, distribution and reproduction in any medium or format, as long as you give appropriate credit to the original author(s) and the source, provide a link to the Creative Commons licence, and indicate if changes were made. The images or other third party material in this article are included in the article's Creative Commons licence, unless indicated otherwise in a credit line to the material. If material is not included in the article's Creative Commons licence and your intended use is not permitted by statutory regulation or exceeds the permitted use, you will need to obtain permission directly from the copyright holder. To view a copy of this licence, visit http://creativecommons.org/licen ses/by/4.0/.

\section{References}

Adams, C. J. (1990). The sexual politics of meat. A feminist-vegetarian critical theory. Bloomsbury.

Adams, M. (2018). The aesthetic value of local food. The Monist, 101(3), 324-339. https://doi.org/10. 1093/monist/ony009

Barnhill, A., King, K. F., Kass, N., \& Faden, R. (2014). The value of unhealthy eating and the ethics of healthy eating policies. Kennedy Institute of Ethics Journal, 24(3), 187-217. https://doi.org/10. 1353/ken.2014.0021

Biltekoff, C. (2013). Eating right in America. Duke University Press.

Biltekoff, C., Mudry, J., Kimura, A. H., Landecker, H., \& Guthman, J. (2014). Interrogating moral and quantification discourses in nutritional knowledge. Gastronomica, 14(3), 17-26. https://doi.org/10. 1525/gfc.2014.14.3.17

Borghini, A. (2014). Geographical indications, food, and culture. In D. M. Kaplan \& P. B. Thompson (Eds.), Encyclopedia of food and agriculture ethics (pp. 1115-1120). Springer.

Borghini, A. (2015). What is a recipe? Journal of Agricultural and Environmental Ethics, 28(4), 719738. https://doi.org/10.1007/s10806-015-9556-9

Borghini, A. (2022). Seven philosophical questions about recipes. In A. Borghini \& P. Engisch (Eds.), A philosophy of recipes. Making, experiencing, and valuing (pp. 15-27). London: Bloomsbury.

Borghini, A., \& Engisch, P. (2022). A philosophy of recipes. Making, experiencing, and valuing. London: Bloomsbury.

\footnotetext{
${ }^{20}$ This research was funded by the Department of Philosophy 'Piero Martinetti' of the University of Milan under the Project 'Department of Excellence 2018-2022' awarded by the Ministry of Education, University and Research (MIUR).
} 
Borghini, A., \& Gandolini, M. (2020). Recipes, their authors, and their names. Humana. Mente, 13(38), 143-162.

Borghini, A., \& Piras, N. (2021). On interpreting something as food. Food Ethics, 6(1). https://doi.org/10. 1007/s41055-020-00082-5

Borghini, A., Piras, N., \& Serini, B. (2020a). Ontological frameworks for food utopias. Rivista Di Estetica, 75, 120-142. https://doi.org/10.4000/estetica.7375.

Borghini, A., Piras, N., \& Serini, B. (2020b). A gradient framework for wild foods. Studies in History and Philosophy of Science Part c: Studies in History and Philosophy of Biological and Biomedical Sciences. https://doi.org/10.1016/j.shpsc.2020.101293

Borghini, A., Piras, N., \& Serini, B. (2021). Eating local. A philosophical toolbox. The Philosophical Quarterly. https://doi.org/10.1093/pq/pqab039.

Brigandt, I., \& Rosario, E. (2020). Strategic conceptual engineering for epistemic and social aims. In A. Burgess, H. Cappelen, \& D. Plunkett (Eds.), Conceptual engineering and conceptual ethics (pp. 100-124). Oxford University Press.

Burgess, A., \& Plunkett, D. (2013). Conceptual ethics I. Philosophy Compass, 8(12), 1091-1101. https:// doi.org/10.1111/phc3.12086

Burgess, A., Cappelen, H., \& Plunkett, D. (Eds.). (2020). Conceptual engineering and conceptual ethics. Oxford University Press.

Cappelen, H. (2018). Fixing language. An essay on conceptual engineering. Oxford University Press.

Cappelen, H., \& Plunkett, D. (2020). Introduction. A Guided Tour Of Conceptual Engineering and Conceptual Ethics. In A. Burgess, H. Cappelen, D. Plunkett (Eds.) Conceptual engineering and conceptual ethics (pp. 1-25). Oxford: Oxford University Press.

Carnap, R. (1950). Empiricism, semantics, and ontology. Revue Internationale De Philosophie, 4(11), 20-40.

Carpenter, K. J. (2003a). A short history of nutritional science: Part 1 (1785-1885). The Journal of Nutrition, 133(3), 638-645. https://doi.org/10.1093/jn/133.3.638

Carpenter, K. J. (2003b). A short history of nutritional science: Part 2 (1885-1912). The Journal of Nutrition, 133(4), 975-984. https://doi.org/10.1093/jn/133.4.975

Carpenter, K. J. (2003c). A Short history of nutritional science: Part 3 (1912-1944). The Journal of Nutrition, 133(10), 3023-3032. https://doi.org/10.1093/jn/133.10.3023

Carpenter, K. J. (2003d). A short history of nutritional science: Part 4 (1945-1985). The Journal of Nutrition, 133(11), 3331-3342. https://doi.org/10.1093/jn/133.11.3331

Counihan, C., \& Van Esterik, P. (Eds.). (2013). Food and culture. A reader. New York and London: Routledge.

Dooley, D., Griffiths, E. J., Gosal, G. S., Buttigieg P. L., Hoehndorf R., Lange M. C., Schriml L. M., Brinkman, F. S. L., \& Hsiao, W. W. L. (2018). FoodOn: A harmonized food ontology to increase global food traceability, quality control and data integration. npj Science of Food 2, article 23. https://doi.org/10.1038/s41538-018-0032-6

Dupré, J., \& O'Malley, M. A. (2009). Varieties of living things: life at the intersection of lineage and metabolism. Philosophy and Theory in Biology, 1, 1-25. https://doi.org/10.3998/ptb.6959004.0001. 003.

Durham, C. A., King, R. P., \& Roheim, C. A. (2009). Consumer definitions of "Locally Grown" for fresh Fruits and vegetables. Journal of Food Distribution Research, 40(1), 56-62. https://doi.org/10. 22004/ag.econ.162116.

FAO (2006). The state of food insecurity in the world 2006. http://www.fao.org/3/a0750e/a0750e00.pdf. Accessed 10 Oct 2020.

Fromartz, S. (2006). Organic, INC. Organic foods and how they grow. Harcourt.

Gallie, W. B. (1955). Essentially contested concepts. Proceedings of the Aristotelian Society, 56, $167-198$.

García, M. E. (2013). The taste of conquest: Colonialism, cosmopolitics, and the dark side of Peru's gastronomic boom. The Journal of Latin American and Caribbean Anthropology, 18(3), 505-524. https://doi.org/10.1111/jlca.12044

García, M. E. (2021). Gastropolitics and the specter of race. Stories of capital, culture, and coloniality in Peru. Oakland: University of California Press.

Giovannucci, D., Josling, T., Kerr, W., O’Connor, B., \& Yeung, M. T. (2009). Guide to geographical indications. Linking products and their origin. International Trade Organization.

Gray, M. (2014). Labor and the locavore. The making of a comprehensive food ethic. University of California Press. 
Guthman, J., Broad, G., Klein, K., \& Landecker, H. (2014). Beyond the Sovereign Body. Gastronomica, 14(3), 46-55. https://doi.org/10.1525/gfc.2014.14.3.46.

Jacobs, D. R., \& Tapsell, L. C. (2007). Food, not nutrients, is the fundamental unit in nutrition. Nutrition Reviews, 65(10), 439-450.https://doi.org/10.1111/j.1753-4887.2007.tb00269.x

Kamminga, H., \& Cunningham, A. (Eds.). (1995). The science and culture of nutrition (1840-1940). Leiden: Brill.

Haslanger, S. (2012). Resisting reality. Oxford University Press.

Hayes-Conroy, J., Hite, A., Klein, K., Biltekoff, C., \& Kimura, A. H. (2014). Doing Nutrition Differently. Gastronomica, 14(3), 56-66. https://doi.org/10.1525/gfc.2014.14.3.56

Ho, H.-T. (2020). Cosmopolitan locavorism: Global local-food movements in postcolonial Hong Kong. Food, Culture \& Society, 23(2), 137-154. https://doi.org/10.1080/15528014.2019.1682886

Holt, G., \& Amilien, V. (2007). Introduction: From local food to localised food. Anthropology of Food, S2, Article S2. https://doi.org/10.4000/aof.405

Klein, K. C. (2013). Ecological nutrition. Redefining healthy food in health care. Ph.D. Dissertation. University of California, Berkeley.

Koch, S. (2020). Engineering what? On concepts in conceptual engineering. Synthese. Online first. https://doi.org/10.1007/s11229-020-02868-w

Landecker, H. (2011). Food as exposure: Nutritional epigenetics and the new metabolism. BioSocieties, 6(2), 167-194. https://doi.org/10.1057/biosoc.2011.1

Landecker, H. (2013a). The metabolism of philosophy, in three parts. In B. Malkmus \& I. Cooper (Eds.). Dialectic and paradox. Configurations of the third in modernity (pp. 193-224). Bern: Peter Lang.

Landecker, H. (2013b). Postindustrial metabolism: Fat knowledge. Public Culture, 25(3), 495-522. https://doi.org/10.1215/08992363-2144625

Lien, M. E. (2015). Becoming a salmon. Aquaculture and the domestication of a fish. University of California Press.

Manne, K. (2018). Down girl. The logic of misogyny. Oxford University Press.

McClements, D. J., Vega, C., Mcbride, A., \& Decker, E. A. (2011). Defense of Food Science. Gastronomica, 11(2), 76-84. https://doi.org/10.1525/gfc.2011.11.2.76.

McWilliams, J. E. (2010). Just food: Where locavores get it wrong and how we can truly Eat Responsibly. Back Bay Books.

Montanari, M. (2006). Food is culture. Columbia University Press.

Mudry, J. (2009). Measured meals. Nutrition in America. SUNY Press.

Mueller, T. (2011). Extra virginity: The sublime and scandalous world of olive oil. W. W. Norton \& Company.

Murphy, D. (2020). Concepts of disease and health. In E. N. Zalta (Ed.), The Stanford Encyclopedia of Philosophy (Summer 2020). Metaphysics Research Lab, Stanford University. https://plato.stanford. edu/archives/sum2020/entries/health-disease/

Nestle, M. (2013). Food politics. How the food industry influences nutrition and health, Revised and expanded tenth (anniversary). University of California Press.

Paxson, H. (2010). Locating value in artisan cheese: Reverse engineering terroir for new-world landscapes. American Anthropologist, 112(3), 444-457. https://doi.org/10.1111/j.1548-1433.2010. 01251.x

Pogge, T. (2016). The hunger games. Food. Ethics, 1(1), 9-27. https://doi.org/10.1007/s41055-016-0006-9

Pollan, M. (2008). In Defence of food: An eater's manifesto. Penguin.

Powell, A., \& Dupré, J. (2009). From molecules to systems: The importance of looking both ways. Studies in History and Philosophy of Science Part c: Studies in History and Philosophy of Biological and Biomedical Sciences, 40(1), 54-64. https://doi.org/10.1016/j.shpsc.2008.12.007

Raubenheimer, D., Simpson, S. J., \& Mayntz, D. (2009). Nutrition, ecology and nutritional ecology: Toward an integrated framework. Functional Ecology, 23(1), 4-16. https://doi.org/10.1111/j.13652435.2009.01522.x

Scrinis, G. (2008). On the Ideology of Nutritionism. Gastronomica, 8(1), 39-48. https://doi.org/10.1525/ gfc.2008.8.1.39

Scrinis, G. (2013). Nutritionism. The science and politics of dietary advice. Columbia University Press.

Siipi, H. (2013). Is natural food healthy? Journal of Agricultural and Environmental Ethics, 26(4), 797812. https://doi.org/10.1007/s10806-012-9406-y

Siipi, H. (2008). Dimensions of naturalness. Ethics \& the Environment, 13, 71-103. https://doi.org/10. 1353/een.0.0001 
Simopoulos, A. P., \& Visioli, F. (Eds.). (2000). Mediterranean diets. Special issue. World Review of Nutrition and Dietetics, 87.

Simopoulos, A. P. (2001). The Mediterranean diets: What is so special about the diet of Greece? The scientific evidence. The Journal of Nutrition, 131(11), 3065S-3073S. https://doi.org/10.1093/jn/131. 11.3065S

Smith, B. (2001). Fiat objects. Topoi, 20(2), 131-148. https://doi.org/10.1023/A:1017948522031

Smith, A., \& MacKinnon, J. B. (2007). The 100-mile diet: A year of local eating. Random House.

Spence, C. (2017). Gastrophysics. The new science of eating. Penguin.

Ströhle, A., \& Döring, F. (2010). Molecularization in nutritional science: A view from philosophy of science. Molecular Nutrition \& Food Research, 54(10), 1385-1404. https://doi.org/10.1002/mnfr. 201000078

Sylla, N. S. (2014). The fair trade scandal. Marketing poverty to benefit rich. Ohio University Press.

Thomasson, A. L. (2017). Metaphysics and conceptual negotiation. Philosophical Issues, 27(1), 364382.https://doi.org/10.1111/phis.12106

Thompson, P. B. (2010). The agrarian vision. Sustainability and environmental ethics. The University Press of Kentucky.

Tollefsen, D. (2015). Groups as agents. Polity.

Werkheiser, I., \& Noll, S. (2014). From food justice to a tool of the status quo: Three sub-movements within local food. Journal of Agricultural and Environmental Ethics, 27(2), 201-210. https://doi. org/10.1007/s10806-013-9459-6

Yeung, M. T. (2014). Geographic indications. In D. M. Kaplan \& P. B. Thompson (Eds.), Encyclopedia of Food and Agriculture Ethics (pp. 1433-1441). Springer.

Publisher's Note Springer Nature remains neutral with regard to jurisdictional claims in published maps and institutional affiliations. 